\title{
Tomato Fruit Shape Analysis Using Morphometric and Morphology Attributes Implemented in Tomato Analyzer Software Program
}

\author{
Maria Jose Gonzalo \\ Department of Horticulture and Crop Science, The Ohio State University, Wooster, OH 44691 and \\ Dept. de Pomología, Estación Experimental de Aula Dei-CSIC, Apdo. 13034, 50080 Zaragoza, Spain
}

Marin Talbot Brewer ${ }^{1}$, Claire Anderson, and David Sullivan

Department of Horticulture and Crop Science, The Ohio State University, Wooster, OH 44691

\author{
Simon Gray \\ Department of Mathematics and Computer Science, The College of Wooster, Wooster, OH 44691
}

Esther van der Knaap ${ }^{2}$

Department of Horticulture and Crop Science, The Ohio State University, Wooster, OH 44691

AdDitional INDEX words. quantitative trait loci, genetics, molecular markers

\begin{abstract}
Reliable analysis of plant traits depends on the accuracy of scoring the phenotype. We report here on the efficacy of two methods in the detection of quantitative trait loci (QTL) controlling fruit morphology in three segregating tomato (Solanum spp.) $F_{2}$ populations using the software program, Tomato Analyzer. The first method uses fruit morphology attributes such as fruit shape index, blockiness, pear shape, indentation area, and angles of the fruit along the boundary. The second method uses morphometric points to quantify shape. The morphometric data were subjected to principle components analysis (PCA). QTL that control the fruit morphology attributes and the morphometric PCA were identified that revealed that the methods were comparable in that they resulted in nearly identical loci. Novel attributes were added to Tomato Analyzer that improved versatility of the program in measuring additional morphological features of fruit. We demonstrated that these novel attributes permitted identification of QTL controlling the traits.
\end{abstract}

The domestication and breeding of cultivated tomato (Solanum lycopersicum L.) resulted in a diverse collection of varieties that differ in fruit shape and size (Paran and van der Knaap, 2007). Tomato is an excellent model to use to gain insights into the molecular basis of this diversity because of the extensive genetic and genomic resources available for this species. We recently developed a software application, Tomato Analyzer (TA; Van der Knaap et al., 2008), which is rapidly becoming the standard for fruit morphological characterizations (Brewer et al., 2006, 2007; Gonzalo and van der Knaap, 2008). Ontology terms for the parts of the fruit were developed, as well as intuitive mathematical descriptors that quantify many shape features based on the boundary of the fruit. The terms and descriptors were implemented in TA for phenotypic measurements of fruit shape in a semiautomatic and reproducible manner (Brewer et al., 2006). The TA application provides accurate and objective measurements of fruit shape in a highthroughput manner, as well as for traits that are nearly impossible to quantify manually.

The key feature of the software application TA is that a unique mathematical descriptor leads to automated, objective

Received for publication 13 June 2008. Accepted for publication 23 Aug. 2008. This work was supported by NSF-DBI 0227541. Maria Jose Gonzalo was also supported by a fellowship (EX2004-0293) from The Ministry of Education and Science of Spain.

${ }^{1}$ Current address: Department of Plant Pathology and Plant-Microbe Biology, Cornell University, Ithaca, NY 14853.

${ }^{2}$ Corresponding author. E-mail: vanderknaap.1@osu.edu. quantification of the associated shape attribute. Currently, TA features 37 attributes with unique mathematical descriptors. For some of the attributes (e.g., for distal end angles and blockiness of the fruit), the user can select where the measurement is taken. This flexibility allows additional measurements on each object to be taken. Previously, five different progeny populations derived from crosses between $S$. lycopersicum and a close wild relative (Solanum pimpinellifolium L.) accession LA1589 were analyzed for fruit shape using TA (Brewer et al., 2007; Gonzalo and van der Knaap, 2008). The phenotypic output of each attribute was correlated to the genotypic marker information of each plant in the five populations using QTL Cartographer (Wang et al., 2006). These analyses resulted in the identification of numerous shape quantitative trait loci (QTL) distributed across the tomato genome (Brewer et al., 2007; Gonzalo and van der Knaap, 2008).

Shape and size diversity can also be analyzed via morphometric studies, defined as the quantitative analysis of a biological form (Bookstein, 1982; Rohlf, 1990). Morphometric analysis uses the position of and distance between landmarks of the object as the source of morphological data. This method has been used to investigate phenotypic variation for studies in systematic and classification (Chandler and Crisp, 1998; Henderson, 2006; Klingenberg and Monteiro, 2005; Lihova et al., 2004; Sonibare et al., 2004; Weight et al., 2008), as well as evolutionary analyses (Borba et al., 2007; Langlade et al., 2005). Morphometric analysis has also been applied in genetic studies of anatomy in animals and insects (Cheverud, 1996; 
Klingenberg et al., 2001; Weber et al., 1999) or leaf shape in plants (Langlade et al., 2005; Perez-Perez et al., 2002) in a complete and nonredundant fashion (Dryden and Mardia, 1998). Morphometric analyses require neither prior knowledge nor predetermined notions of the shape features that the researcher wants to measure.

In some cases, subtle differences in shape may not be captured by the mathematical descriptors that are the hallmark of TA. Instead, these subtle differences might be captured by morphometric analyses, which, after mapping in segregating populations, could result in the identification of additional fruit morphology QTL. These two methods, morphometrics and attribute analysis, have different and complementary strengths. On the one hand, the morphometric analysis is less biased and more high-throughput than attribute analysis, as it is less dependent on extensive manual manipulations. On the other hand, the phenotypic data resulting from morphometric analysis are abstract quantities that capture shape differences, whereas the data from the TA attributes are intuitive quantities such as angles in degrees and shape indices as ratios that best describe specific features of fruit shape. Fortunately, a novel feature of TA is that it provides both methods in the same application, allowing the user to select the method that is best suits his/her research needs.

One of the major goals of this study was to assess the efficacy of the morphometric analyses and to contrast the QTL identified with morphometrics to the QTL identified with the analyses of specific attributes that were largely based on fruit boundary measurements. Although the boundary-based measurements capture nearly all variation displayed by a fruit, features such as pericarp, placenta, or septum areas could not be measured using the outer circumference of the object. Therefore, a novel set of measurements was added under the "latitudinal section" that calculates the area of the pericarp, septum, and placenta relative to the total fruit area. These novel features described internal morphology aspects that were related to fruit mass.

\section{Materials and Methods}

Plant material. Three $\mathrm{F}_{2}$ populations constructed from crosses between elongated $S$. lycopersicum cultivars (Howard German, Sausage, and Rio Grande) and the wild species $S$. pimpinellifolium accession LA1589 carrying round fruit weighing $\approx 1 \mathrm{~g}$ were used in the analyses. The 'Howard German' $\mathrm{F}_{2}$ $\left(\mathrm{HGF}_{2}\right)$ population, containing 130 plants, was reported by Brewer et al. (2007). The 'Sausage' $F_{2}$ population $\left(\mathrm{SAF}_{2}\right)$ consisting of 106 plants and the 94 plants constituting the 'Rio Grande' $\mathrm{F}_{2}$ population $\left(\mathrm{RGF}_{2}\right)$ were reported by Gonzalo and van der Knaap (2008). The fourth population $\left(\mathrm{HGBC}_{1} \mathrm{~F}_{2}\right)$ with 89 plants was obtained by selfing a 'Howard German' Backcross population $\left(\mathrm{HGBC}_{1}\right)$, which was reported previously (Brewer et al., 2007). In general, eight fruit per plant were analyzed.

Phenotypic Analysis. Fruit scanning, as well as the manual adjustments and morphological analyses by TA, was previously reported (Brewer et al., 2006). Herein, we only discuss the attributes that were added or modified in TA. The new version, TA 2.2, is available with manual from Van der Knaap et al. (2008).

MorPhOMETRIC ANALYSES. The "morphometrics" function set of TA uses the distal and proximal ends as landmark points for every object, as shown in Fig. 1A. The number of points found along the boundary is user-defined, from 4 to 30 . These points will be evenly spaced between the distal and proximal ends. The first point $(\mathrm{x} 1, \mathrm{y} 1)$ is the proximal endpoint and the following points move counter-clockwise around the object. The lowest " $\mathrm{x}$ " coordinate is at the far left, whereas the lowest " $y$ " coordinate is at the far top, resulting in positive values for the morphometric coordinates. The batch analysis assigns
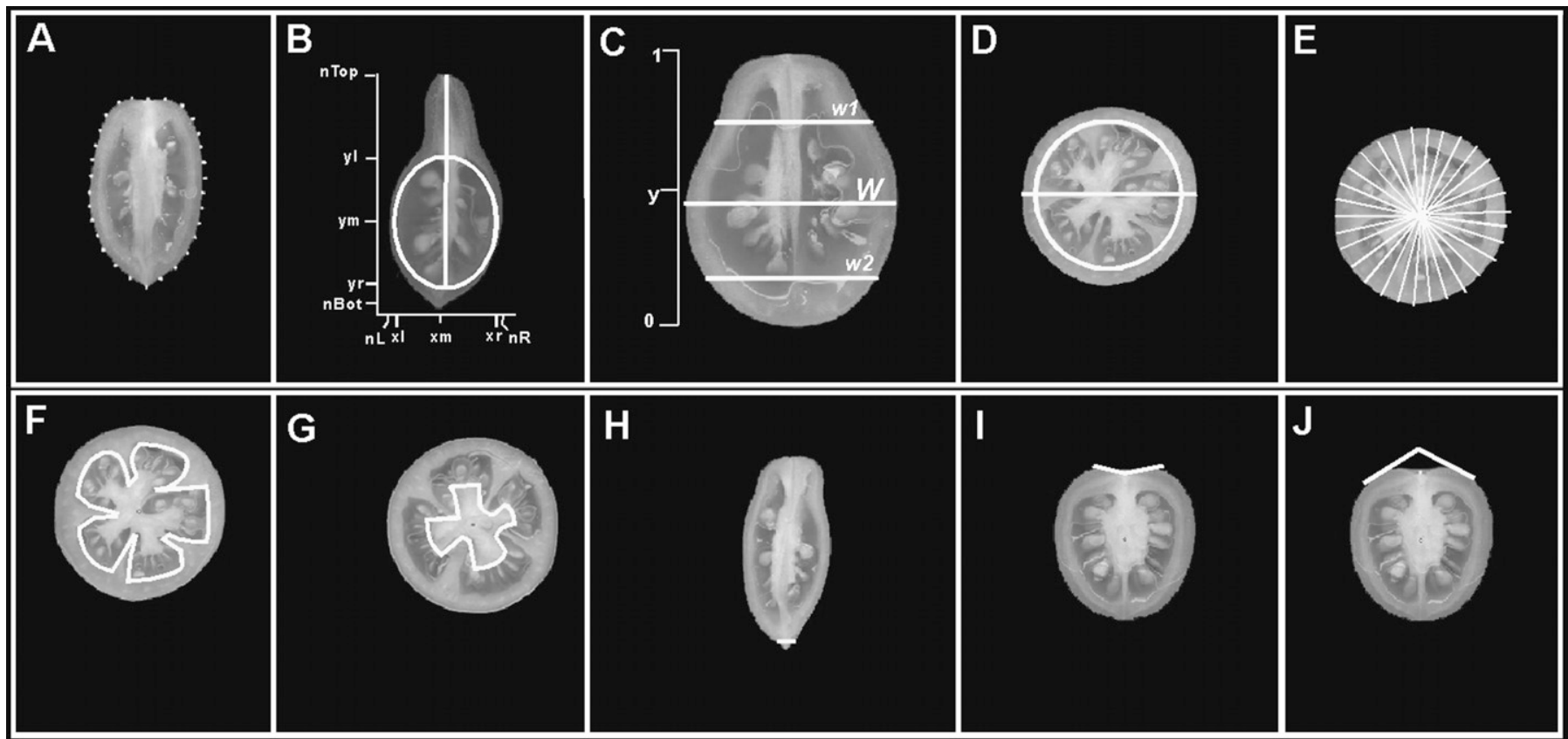

Fig. 1. Descriptors of fruit morphology attributes. (A) Morphometrics, (B) internal eccentricity, (C) obovoid and widest width position, (D) pericarp area, (E) lobedness degree, $(\mathbf{F})$ pericarp and septum area, $(\mathbf{G})$ placenta area, $(\mathbf{H})$ distal end protrusion, $(\mathbf{I})$ proximal end angle at $2 \%$, and $(\mathbf{J})$ proximal end angle at $20 \%$. 

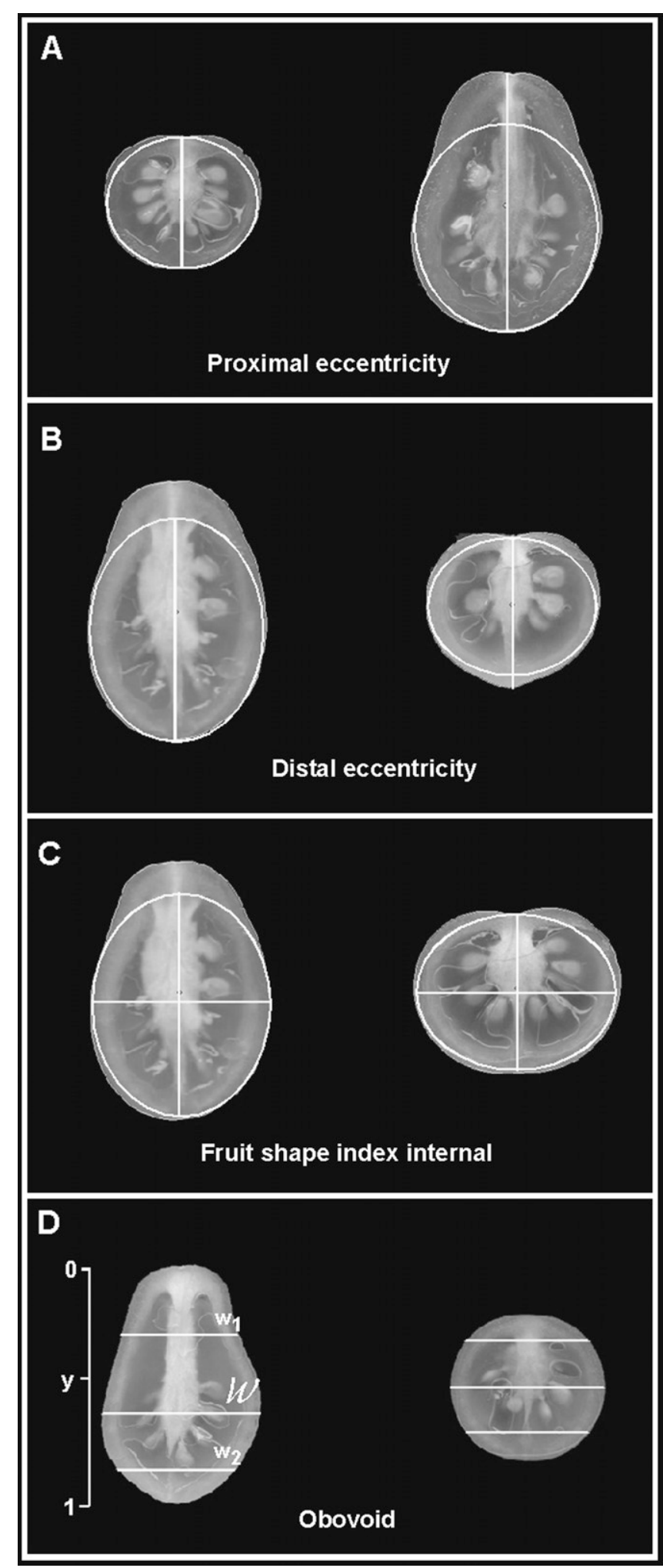

Fig. 2. Eccentricity and asymmetry attributes sorted from the highest (left) to the lowest (right) value. Fruit were analyzed from tomato plants in the 'Sausage' $F_{2}$ population. (A) Proximal eccentricity: The proximal eccentricity for the round fruit is close to 1 , whereas for the pear-shaped fruit, this value is less than 1 , at 0.81. (B) Distal eccentricity: For the round bottom fruit on the left, the distal eccentricity is close to 1 , whereas for the slightly pointed fruit, the value is less than 1, at 0.91. (C) Fruit shape index internal: For the fruit on the left, the shape index internal is 1.26, whereas the index for the fruit on the right is 0.78 . (D) Obovoid: Fruit with the highest obovoid value of 0.19 is shown on the right, whereas the obovoid value for round and ovoid fruit is returned as " 0. " average coordinate values for each point along the boundary of the objects for each image.

INTERNAL ECCENTRICITY. Under the new "internal eccentricity" function set, a collection of attributes was implemented to calculate the degree of eccentricity of the seed position in the fruit. First, an ellipse is manually drawn around the seed area. TA scans the ellipse to determine its horizontal and vertical axes, yl-yr and xl-xr, respectively, as shown in Fig. 1B. Proximal eccentricity is measured as the degree of eccentricity at the proximal end of the fruit. It is defined as the ratio between the vertical axis of the ellipse and the distance from the bottom of the ellipse to the top of the fruit.

$$
(\mathrm{yr}-\mathrm{yl}) /(\mathrm{yr}-\mathrm{nTop})
$$

Distal eccentricity is measured as the ratio between the vertical axis of the ellipse and the distance from the top of the ellipse or circle to the bottom of the fruit.

$$
(\mathrm{yr}-\mathrm{yl}) /(\text { nBot }-\mathrm{yl})
$$

Eccentricity is measured as the ratio between the vertical axis and the total fruit height.

$$
(\mathrm{yr}-\mathrm{yl}) /(\mathrm{nBot}-\mathrm{nTop})
$$

The internal shape index (also known as pericarp elongation index) is calculated as the ratio of vertical and horizontal axes of the ellipse or circle.

$$
(\mathrm{yr}-\mathrm{yl}) /(\mathrm{xr}-\mathrm{xl})
$$

The eccentricity area index function under the "internal eccentricity" function set is measured as the ratio of the area of the ellipse and the total fruit area.

Asymmetry. For fruit "asymmetry," one attribute was added while others were improved. We improved the original obovoid and ovoid attributes, which describe the degree to which the fruit is bottom or top heavy, respectively, with a more accurate mathematical descriptor. For obovoid, the fruit must follow one criterion, as shown in Fig. 1C: the area below the midpoint must be larger than the area above the midpoint. If this is not the case, the value is 0 . Otherwise, the two criteria, position of the maximum width (W) and the taperness of the fruit, are calculated (Brewer et al., 2006).

$$
\mathrm{T}=1-\mathrm{w} 1 / \mathrm{W}+\mathrm{w} 2 / \mathrm{W}
$$

The following formula is then used to calculate obovoid.

$$
\text { Obovoid }=1 / 2 \times f(W) T
$$

The converse is true for ovoid: when the area below the midpoint is smaller than the area above the midpoint, the other two criteria will be calculated and returned as a value for ovoid. The new feature added is the position of the maximum width of the fruit along the longitudinal axis (W in Fig. 1C) The software assigns a value from 0 to 1 depending on the distance of the widest width from the proximal end of the fruit, with values approaching 1 at positions closest to the distal end.

LATITUDinAL SECTION. The degree of uneven shape of the fruit, lobedness, is reflected by the standard deviation of the lengths between each boundary point and the weight center, as shown in Fig. 1E. Locule number was determined visually because it was not possible to accurately automate this task in TA. 


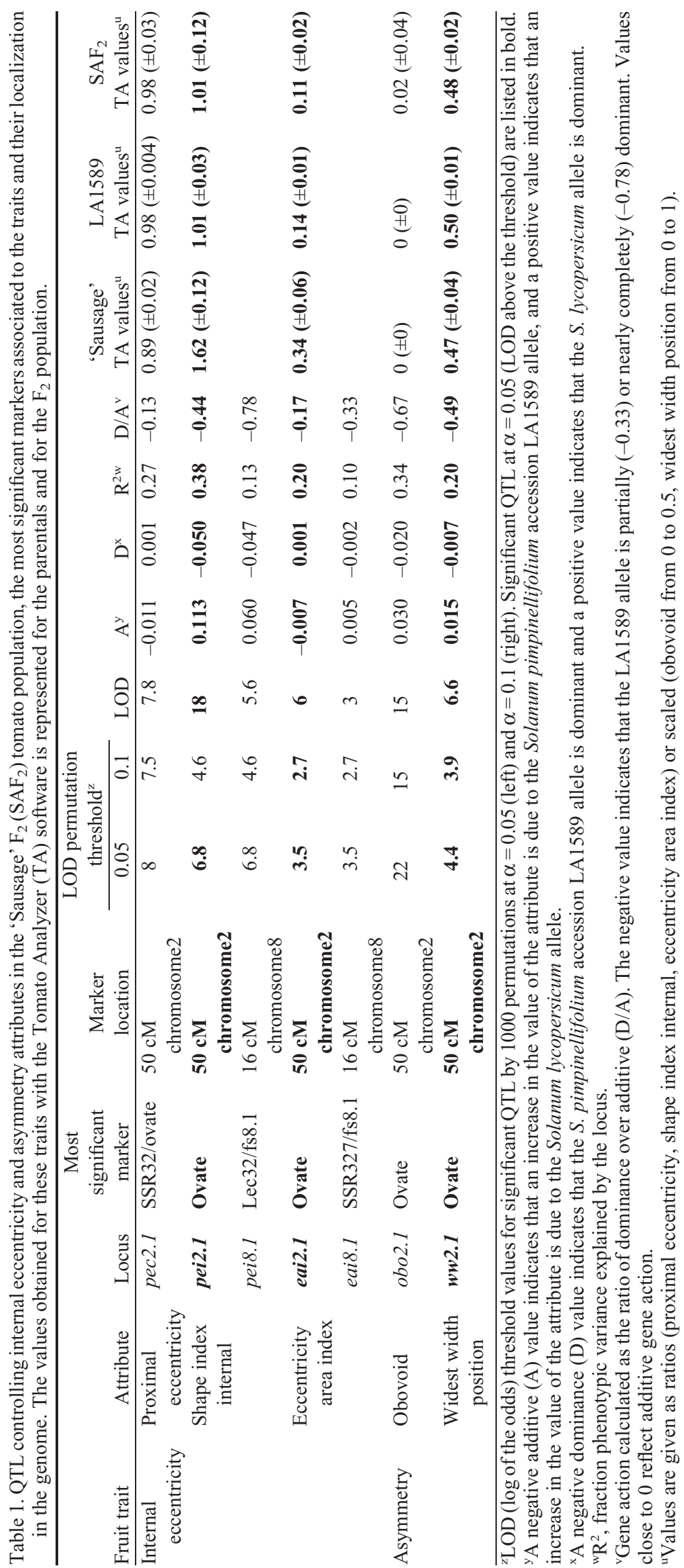


Table 2. QTL controlling proximal angle in the three tomato $F_{2}$ populations, the most significant marker associated with the trait and their position.

\begin{tabular}{|c|c|c|c|c|c|c|c|c|c|c|c|c|}
\hline $\mathrm{F}_{2}$ population & $\begin{array}{c}\text { Trait } \\
\text { category }\end{array}$ & Attribute $^{z}$ & Locus & $\begin{array}{c}\text { Most } \\
\text { significant } \\
\text { marker } \\
\end{array}$ & Marker location & $\begin{array}{c}\text { LOD } \\
\text { permutation } \\
\text { threshold }^{\mathrm{y}}\end{array}$ & LOD & $A^{x}$ & $\mathrm{D}^{\mathrm{w}}$ & $\mathrm{R}^{2 \mathrm{v}}$ & $\mathrm{D} / \mathrm{A}^{\mathrm{u}}$ & $\begin{array}{c}\text { Previous } \\
\text { results }^{t}\end{array}$ \\
\hline \multirow[t]{5}{*}{$\begin{array}{l}\text { 'Howard } \\
\text { German' }\end{array}$} & $\begin{array}{c}\text { Proximal } \\
\text { end }\end{array}$ & angle2 & pan7.2 & sun & $27 \mathrm{cM}$ chromosome7 & 3.3 & 6.5 & -5.58 & 0.66 & 0.19 & -0.12 & pan7.2 \\
\hline & & & pan 7.3 & SSR45 & $88 \mathrm{cM}$ chromosome7 & & 5.6 & 5.69 & -0.19 & 0.18 & -0.03 & pan 7.3 \\
\hline & & angle5 & pan 7.2 & sun & $27 \mathrm{cM}$ chromosome 7 & 4.8 & 7.6 & -8.87 & -2.00 & 0.23 & 0.23 & pan 7.2 \\
\hline & & angle 10 & pan 7.2 & sun & $27 \mathrm{cM}$ chromosome 7 & 4.3 & 26.7 & -17.50 & -0.66 & 0.58 & 0.04 & pan 7.2 \\
\hline & & angle 20 & pan 7.2 & sun & $27 \mathrm{cM}$ chromosome 7 & 4.5 & 36.8 & -26.68 & 0.14 & 0.70 & -0.01 & pan 7.2 \\
\hline \multirow[t]{7}{*}{ 'Sausage' } & $\begin{array}{l}\text { Proximal } \\
\text { end }\end{array}$ & angle2 & pan1.1 & TG273 & $50 \mathrm{cM}$ chromosome 1 & 3.4 & 3.5 & 5.05 & 1.47 & 0.13 & 0.29 & pan 1.1 \\
\hline & & & pan 2.1 & ovate & $50 \mathrm{cM}$ chromosome2 & & 5.3 & -5.89 & 0.39 & 0.16 & -0.07 & pan 2.1 \\
\hline & & angle5 & pan 1.1 & TG273 & $50 \mathrm{cM}$ chromosome1 & 3.3 & 3.5 & 6.30 & -2.94 & 0.11 & -0.47 & pan1.1 \\
\hline & & & pan 2.1 & ovate & $50 \mathrm{cM}$ chromosome2 & & 9.2 & -10.29 & 4.87 & 0.27 & -0.47 & pan 2.1 \\
\hline & & angle 10 & pan 2.1 & ovate & $50 \mathrm{cM}$ chromosome2 & 3.7 & 16.0 & -13.07 & 7.62 & 0.41 & -0.58 & pan 2.1 \\
\hline & & angle 20 & pan 2.1 & ovate & $50 \mathrm{cM}$ chromosome2 & 4.5 & 15.5 & -14.12 & 8.17 & 0.35 & -0.58 & pan 2.1 \\
\hline & & & pan8.1 & SSR327/fs8.1 & $16 \mathrm{cM}$ chromosome 8 & & 4.6 & -7.52 & 6.46 & 0.11 & -0.86 & \\
\hline \multirow[t]{3}{*}{ 'Rio Grande' } & Proximal & angle2 & pan 3.2 & TG134 & $97 \mathrm{cM}$ chromosome 3 & 3.8 & 8.2 & 5.96 & 0.95 & 0.30 & 0.16 & pan3.2 \\
\hline & & $\begin{array}{l}\text { angle5 } \\
\text { angle10 }\end{array}$ & & & & & & & & & & \\
\hline & & angle 20 & pan1.1 & SSR192 & $37 \mathrm{cM}$ chromosome1 & 3.5 & 4.0 & 4.66 & -1.88 & 0.13 & -0.40 & \\
\hline
\end{tabular}

${ }^{\mathrm{z}}$ Attributes associated with a number indicate the position along the boundary at which the slope was calculated. For example, $2=2 \%$ from the proximal end.

${ }^{y}$ LOD (log of the odds) threshold values for significant QTL by 1000 permutations at $\alpha=0.05$.

${ }^{\mathrm{x}} \mathrm{A}$ negative additive (A) value indicates that an increase in the value of the attribute is due to the Solanum pimpinellifolium accession LA1589 allele, and a positive value indicates that an increase in the value of the attribute is due to the Solanum lycopersicum allele.

${ }^{\text {w}}$ A negative dominance (D) value indicates that the $S$. pimpinellifolium accession LA1589 allele is dominant and a positive value indicates that the $S$. lycopersicum allele is dominant.

${ }^{\mathrm{v}} \mathrm{R}^{2}$, fraction phenotypic variance explained by the locus.

"Gene action calculated as the ratio of dominance over additive (D/A). The negative value indicates that the LA1589 allele is partially or nearly completely $(-0.86)$ dominant. Values close to 0 reflect additive gene action.

${ }^{t}$ QTL controlling proximal end angle using a different mathematical descriptor (Gonzalo and van der Knaap, 2008).

Table 3. Mean degrees and standard deviation of the angles for the parental and the individuals of the three tomato $F_{2}$ populations for the proximal fruit end angles.

\begin{tabular}{|c|c|c|c|c|c|c|c|c|}
\hline Trait category & Attribute $^{z}$ & $\mathrm{HG}^{\mathrm{y}}$ & $\mathrm{HGF}_{2}^{\mathrm{y}}$ & $\mathrm{SA}^{\mathrm{y}}$ & $\mathrm{SAF}_{2}^{\mathrm{y}}$ & $\mathrm{RG}^{\mathrm{y}}$ & $\mathrm{RGF}_{2}^{\mathrm{y}}$ & $\mathrm{LA}^{\mathrm{y}}$ \\
\hline \multirow{3}{*}{$\begin{array}{l}\text { Proximal } \\
\text { fruit end }\end{array}$} & angle 2 & $223( \pm 31)$ & $188( \pm 9)$ & $211( \pm 5)$ & $191( \pm 9)$ & $204( \pm 10)$ & $190( \pm 7)$ & $176( \pm 8)$ \\
\hline & angle 5 & $213( \pm 22)$ & $185( \pm 13)$ & $213( \pm 9)$ & $185( \pm 13)$ & $214( \pm 10)$ & $187( \pm 9)$ & $166( \pm 9)$ \\
\hline & angle 20 & $86( \pm 85)$ & $103( \pm 21)$ & $61( \pm 16)$ & $112( \pm 15)$ & $88( \pm 1)$ & $122( \pm 8)$ & $112( \pm 4)$ \\
\hline
\end{tabular}

${ }^{\mathrm{z}}$ Trait acronyms associated with a number indicate the position along the boundary at which the slope was calculated. For example, $2=2 \%$ from the proximal end. The data represent degrees + or - the SD.

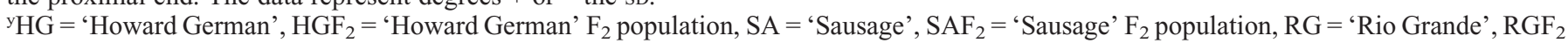
$=$ 'Rio Grande' $\mathrm{F}_{2}$ population, LA = Solanum pimpinellifolium accession LA1589.

To determine variation in the thickness and area of the pericarp, septum, and placenta, we developed a new set of attributes (Fig. 1, D-G), which are listed under the "latitudinal section" function set in TA. The calculation of these fruit areas is based on a user-defined internally shaped boundary. First, for each object, the user selects a default pericarp boundary size that is at a percentage of the external boundary. For example, a default at $80 \%$ results in an inner ellipse with the shape of the outer boundary at $80 \%$ of its size. Subsequently, this ellipse can be manually adjusted to border any area within the fruit such as pericarp (Fig. 1D), pericarp plus septum (Fig. 1F), or placenta area (Fig. 1G). The area between the external and the pericarp boundary is divided over the total fruit area to obtain the pericarp, septum, and placenta area features. Note: the largest placenta will return the smallest value because of the method used to measure the trait. The pericarp thickness values are averaged over four equally distributed measurements that calculate the distance between the outer and the inner boundary.

Distal END Protrusion. The distal end protrusion in the "distal fruit end shape" function set of TA calculates the area of the protruded end multiplied by 10 , over the total fruit area (Fig. 1H). The tip is found by advancing along the boundary of the fruit toward the tip a few points at a time and calculating the slope by using the current point and the previous 
Table 4. QTL analyses of the first three principal components (PC) identified by the morphometric analysis in each of the three tomato $\mathrm{F}_{2}$ populations.

\begin{tabular}{|c|c|c|c|c|c|c|c|c|c|c|}
\hline $\begin{array}{l}\mathrm{F}_{2} \\
\text { population }\end{array}$ & Locus $^{\mathrm{z}}$ & $\begin{array}{c}\text { Most } \\
\text { significant } \\
\text { marker } \\
\end{array}$ & $\begin{array}{l}\text { Marker } \\
\text { location }\end{array}$ & $\begin{array}{c}\text { LOD } \\
\text { permutation } \\
\text { threshold }^{\mathrm{y}}\end{array}$ & LOD & $\mathrm{A}^{\mathrm{x}}$ & $\mathrm{D}^{\mathrm{w}}$ & $\mathrm{R}^{2 \mathrm{v}}$ & $\mathrm{D} / \mathrm{A}^{\mathrm{u}}$ & $\begin{array}{c}\text { Fruit } \\
\text { morphology QTL }^{\mathrm{t}}\end{array}$ \\
\hline \multirow[t]{6}{*}{$\begin{array}{l}\text { 'Howard } \\
\text { German' }\end{array}$} & $p c 1.2$ & TG151 & $105 \mathrm{cM}$ chromosome 2 & 3.5 & 6.0 & 2.88 & -2.08 & 0.16 & -0.72 & $\begin{array}{l}\text { hrt2.1, pblk2.1, pan } 2.1 \text {, } \\
\quad \text { per2.1, ar2.1 }\end{array}$ \\
\hline & pc1.3 & T0581 & $93 \mathrm{cM}$ chromosome 3 & 3.5 & 3.8 & 2.97 & 0.67 & 0.10 & 0.23 & dan3.1, per3.1, ar3.1 \\
\hline & pc1.9 & TG551 & $49 \mathrm{cM}$ chromosome 9 & 3.5 & 3.6 & 2.48 & -0.85 & 0.09 & -0.34 & \\
\hline & pc1.10 & T0787 & $10 \mathrm{cM}$ chromosome 10 & 3.5 & 4.0 & 2.73 & 0.02 & 0.10 & 0.01 & per10.1 \\
\hline & $p c 2.7$ & sun & $30 \mathrm{cM}$ chromosome 7 & 3.6 & 26.4 & -5.77 & 0.48 & 0.59 & -0.08 & $\begin{array}{c}f_{s} 7.1, \text { hrt7.2, dblk7.1, dan } 7.2, \\
\text { psh7.1, pan7.2, piar7.1 }\end{array}$ \\
\hline & pc2.8 & SSR344/fs8.1 & $14 \mathrm{cM}$ chromosome 8 & 3.6 & 5.2 & -1.47 & 1.41 & 0.08 & -0.96 & fs 8.1 , dan 8.1 \\
\hline \multirow[t]{5}{*}{ 'Sausage' } & $p c 1.2$ & TG337 & $57 \mathrm{cM}$ chromosome 2 & 3.5 & 4.7 & 3.28 & -1.12 & 0.13 & -0.34 & $\begin{array}{l}f s 2.1, \text { hrt } 2.1, \text { dblk2.1, dan } 2.1, \\
\text { pblk2.1, psh2.1, pan2.1, } \\
\text { piar2.1, per } 2.1, \text { ar } 2.1\end{array}$ \\
\hline & pc1.11 & TG36 & $88 \mathrm{cM}$ chromosome 11 & 3.5 & 7.8 & 4.86 & -0.21 & 0.26 & -0.04 & $\begin{array}{l}\text { dblk11.1, per11.1, } \\
\quad \operatorname{ar11.1,~fw11.3~}\end{array}$ \\
\hline & $p c 2.1$ & TG125 & $37 \mathrm{cM}$ chromosome1 & 4.1 & 4.0 & -1.66 & 0.95 & 0.09 & -0.57 & $\begin{array}{l}\text { tri1.1, hrt1.1, pblk1.1, psh1.1, } \\
\text { pan 1.1, piar1.1, fw1.1 }\end{array}$ \\
\hline & $p c 2.2$ & ovate & $50 \mathrm{cM}$ chromosome 2 & 4.1 & 14.9 & 3.52 & -1.78 & 0.35 & -0.51 & $\begin{array}{l}f_{s} 2.1, \text { hrt } 2.1, \text { dblk2.1, dan } 2.1, \\
\text { pblk2.1, psh2.1, pan2.1, } \\
\text { piar2.1, per } 2.1, \text { ar } 2.1\end{array}$ \\
\hline & pc2.8 & SSR327/fs8.1 & $16 \mathrm{cM}$ chromosome 8 & 4.1 & 3.8 & 1.98 & -0.62 & 0.09 & -0.31 & dan 8.1 \\
\hline \multirow{4}{*}{$\begin{array}{l}\text { 'Rio } \\
\text { Grande' }\end{array}$} & pc1.3.1 & SSR111 & $70 \mathrm{cM}$ chromosome 3 & 3.4 & 6.8 & 4.57 & -0.99 & 0.18 & -0.22 & pan 3.1 \\
\hline & $p c 1.3 .2$ & T0581 & $108 \mathrm{cM}$ chromosome 3 & 3.4 & 4.5 & 3.70 & -0.05 & 0.13 & -0.01 & $\begin{array}{l}\text { tri3.1, hrt3.1, pblk3.1, psh3.1, } \\
\quad \text { pan3.2, per3.1, ar3.1, fw3.1 }\end{array}$ \\
\hline & pc1.11 & TG36 & $84 \mathrm{cM}$ chromosome11 & 3.4 & 4.9 & 2.72 & 2.65 & 0.12 & 0.97 & $\begin{array}{l}\text { pblk11.1, per11.1, } \\
\quad \text { ar11.1, fw11.1 }\end{array}$ \\
\hline & pc3.8 & SSR327/fs8.1 & $30 \mathrm{cM}$ chromosome 8 & 3.3 & 3.5 & 1.62 & -1.08 & 0.21 & -0.67 & $f_{s} 8.1$, dblk8.1, dan 8.1 \\
\hline
\end{tabular}

${ }^{\mathrm{z} Q T L}$ acronyms for principle component (PC) reflect the PC for which it was detected (first number) and the chromosome where it was located (second number).

${ }^{y}$ LOD threshold values for significant QTL by 1000 permutations at $\alpha=0.05$.

${ }^{\mathrm{x}} \mathrm{A}$ negative value indicates that an increase in the value of the attribute is due to the Solanum pimpinellifolium allele, and a positive value indicates that an increase in the value of the attribute is due to the Solanum lycopersicum allele.

${ }^{\text {w}}$ A negative value indicates that the $S$. pimpinellifolium allele is dominant and a positive value indicates that the $S$. lycopersicum allele is dominant.

${ }^{\mathrm{v}} \mathrm{R}^{2}$, fraction phenotypic variance explained by the locus.

"Gene action calculated as the ratio of dominance over additive (D/A). The negative value indicates that the LA1589 allele is partially or nearly completely dominant.

${ }^{t}$ QTLs controlling shape and size attributes detected in previous studies in the same populations (Gonzalo and van der Knaap, 2008).

point. The algorithm looks for an abrupt change in slope between adjacent sets of points, indicating that a tip has been found. The tip can also be adjusted manually by the user. The software assigns the value zero to fruit without a distal end protrusion.

Proximal End Angle. The feature for proximal end angle in the "proximal fruit end shape" function set of TA is now measured similarly to the distal end angle (Brewer et al., 2006). The angle is measured at the intersection of two lines where the slope is measured via regression along the boundary of the fruit on both sides at a user-defined distance from the proximal end of the fruit (Fig. 1, I and J). The slope is determined by the regression measured at $\pm 5 \%$ when the user-defined position is between 5\% and 40\% from the proximal end (macro setting), whereas the slope is determined by the regression measured at $\pm 2 \%$ when the user-defined position is between $2 \%$ and $10 \%$ from the proximal end (micro setting). The angle is measured at the point where the lines intersect and is expressed in degrees, where 180 is flat, greater than 180 indented (Fig. 1I), and less than 180 is tapered (Fig. 1J).
USER SETTINGS AND IMAGE ANALYSIS. TA can save settings for future use, as well as import previously assigned settings. This feature can be found under "setting," in "user settings" on the Menu bar. Included are settings for blockiness, angles, and scanning resolution.

When analyzing multiple images, TA asks the user to select which batch output is desired. Depending on the planned statistical analysis of the TA data, the user can select the "average only" command to obtain the average value for each selected attribute across all objects in each image; the "average and standard deviation" command to obtain the averages described above and the standard deviation; or "individual measurements" to obtain the values for each selected attribute for each object in the image.

After the first selection of the manual adjustment on the drop down menu of the "revise" function set (e.g., boundary, distal end), repeated manual adjustments for the same feature on the same or other objects can be completed by clicking directly on the "revise" button. 


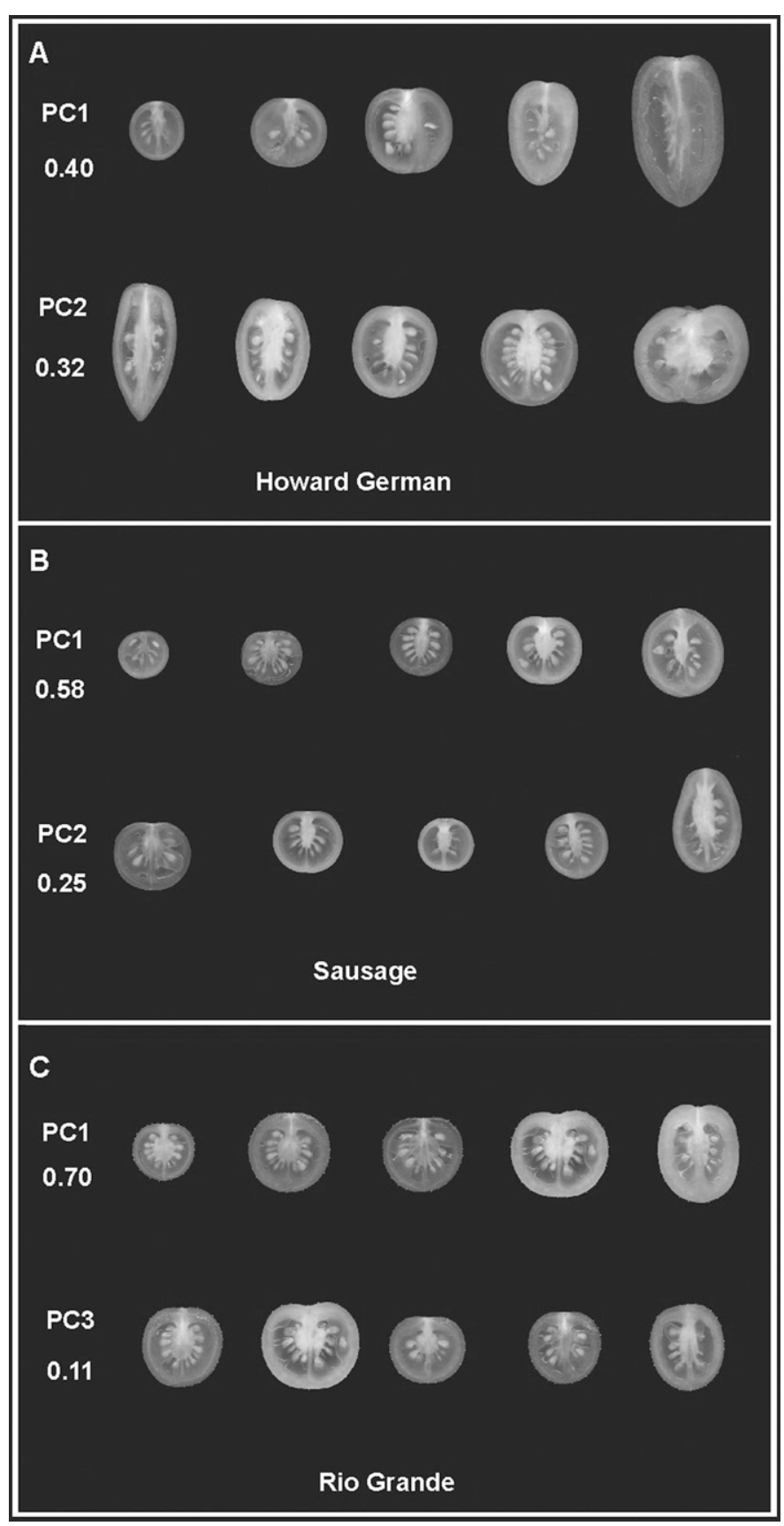

Fig. 3. Tomato fruit sorting based on the morphometric analysis coupled with principal components analysis. (A) Principle component (PC) 1 and PC2 of the 'Howard German' $F_{2}$ population. (B) PC1 and PC2 of the 'Sausage' $F_{2}$ population. (C) $\mathrm{PC} 1$ and $\mathrm{PC} 3$ of the 'Rio Grande' $\mathrm{F}_{2}$ population. The fruit are sorted from low (left) to high (right) values exhibited by the PCs.

STATistical ANALYSis. QTL analysis was performed by composite interval mapping (Zeng, 1993, 1994) using model six with five marker cofactors selected by forward regression and a 10-cM window size, as implemented in Windows QTL Cartographer, version 2.5 (Wang et al., 2006). Permutation tests were conducted 1000 times at a significance level of 0.05 to determine QTL threshold levels (Churchill and Doerge, 1994). QTLs above the significance threshold determined by the permutation tests were considered significant. Additive and dominance effects and the percentage of phenotypic variance explained by the QTL $\left(\mathrm{R}^{2}\right)$ were estimated using Windows QTL Cartographer at highest probability peaks. Dominance is calculated as $\mathrm{D}=\mathrm{Aa}-(\mathrm{AA}+\mathrm{aa}) / 2$, whereas additivity is calculated as $\mathrm{A}=(\mathrm{AA}-\mathrm{aa}) / 2$. Gene action is calculated as $\mathrm{D} / \mathrm{A}$, where " 0 " means additive action, " -1 " or " 1 " dominance of one or the other allele, and values larger than 1 or smaller than -1 mean overdominance.

For the morphometric analyses, the same fruit images were used as for the attribute analyses. The average coordinate values are imported into SAS (version 9; SAS Institute Inc., Cary, NC) and used to create a correlation matrix from which the eigenvectors of the principal components (PC) are calculated. The principle component analysis (PCA) gives a PC value for each individual of the population, which were defined with the "input" command followed by the name of the variables "x1 y1 x2 y2 x3 y3 ...xn yn", where $n$ is the number of points selected for morphometric analysis. These PC values are subsequently used as traits in the QTL analysis. The PCA was conducted by the command "proc princomp" using SAS. The results include the simple statistics (mean and standard deviation of each coordinate), correlation matrix, and the eigenvectors for each PC.

The association of molecular markers and the "latitudinal section" attributes was performed by analysis of variance (ANOVA) using SAS. The genotypes at each marker $(1=$ homozygous for the 'Howard German' parent, 2 = heterozygous, 3 = homozygous for the LA1589) were the grouping variables and the trait attribute values were the dependent variables. The significance of the markers was at the $95 \%, 99 \%$, and $99.9 \%$ confidence level.

\section{Results and Discussion}

We previously demonstrated the utility of the TA software application in assessing tomato fruit shape variation in segregating populations (Brewer et al., 2006, 2007; Gonzalo and van der Knaap, 2008). For each attribute, one mathematical descriptor quantified the feature that was subsequently mapped as a QTL in the population. For this study, and before the comparisons with morphometrics, we expanded TA's tool kit by adding novel attributes, as well as improving existing ones.

IMPLEMENTATION AND EFFICACY ANALYSIS OF NEW AND IMPROVED ATTRIBUTES. The first release of TA (version 2.1) allowed the user to measure fruit elongation by calculating the shape index, which is the ratio of height to width. Pear-shaped fruit differ from oval- and pointy-shaped fruit despite similar fruit shape indices. To distinguish between oval, pear, and pointed fruit, we implemented a set of novel features in TA that were aimed at measuring the degree of eccentric fruit and fall under the "internal eccentricity" function set. Previously developed mathematical descriptors to calculate eccentricity (Van der Knaap et al., 2002) were implemented in TA. An internal ellipse was manually drawn around the seed area using tools in TA (Fig. 2, A-C). Calculations were made based on this ellipse and the boundary of the fruit. A value for proximal eccentricity closer to 1 corresponded to round fruits, whereas a value lower than 1 corresponded to a pear-shaped fruit (Fig. 2A). Similarly, a value for distal eccentricity closer to 1 corresponded to a fruit with a round distal fruit end shape, whereas a value lower than 1 corresponded to a pointed fruit (Fig. 2B). The fruit shape index internal describes the shape of the internal ellipse drawn around the seed area. The synonym 

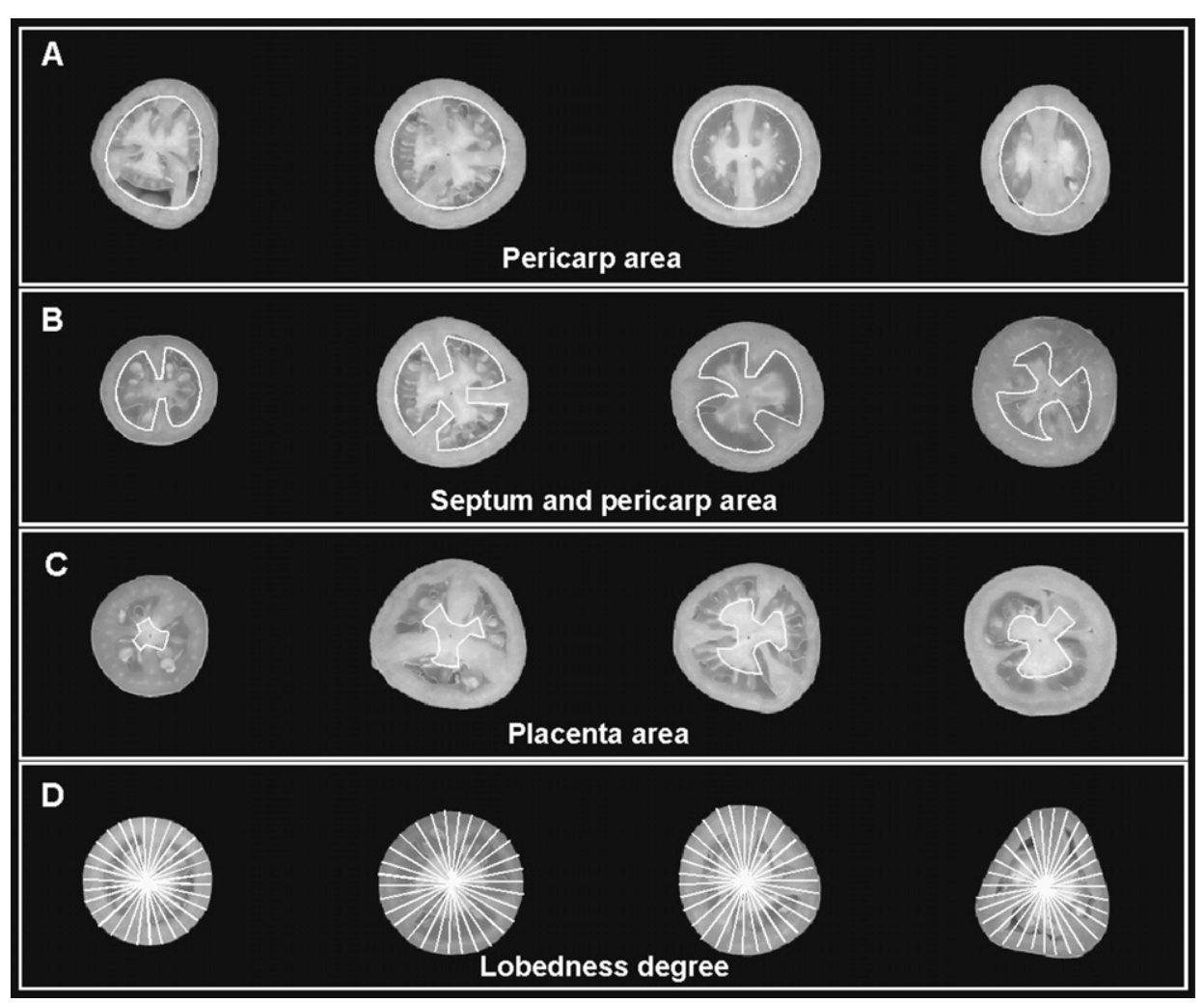

Fig. 4. Latitudinal attributes sorted from the lowest (left) to the highest (right) value. The fruit shown were from tomato plants in the 'Howard German' backcross ${ }_{1} F_{2}$ population. (A) Pericarp area: The lowest pericarp area value is 0.3, whereas the greatest value is approximating 0.5. (B) Pericarp and septum area: The lowest value is 0.4, whereas the greatest value is 0.7. (C) Placenta area: The smaller placentas are on the left featuring a value of 0.95 , whereas the greater placentas are valued at 0.8. (D) Lobedness degree: The value for the round fruit is at 0.6 , whereas the most lobed fruit is at 3.5.

for this attribute is pericarp elongation index. Oval-shaped seed areas will have an internal shape index greater than 1 , whereas round-shaped seed areas will have an index of $\approx 1$ (Fig. 2C). The ratio of the ellipse area over total fruit area is described by the eccentricity area index. The data obtained with TA for these attributes were used to perform QTL analysis in the $\mathrm{SAF}_{2}$ population because it was segregating for ovate, a locus controlling elongated and pear-shaped fruit. Although we detected other QTL with a log of the odds (LOD) below the threshold level at ovate and $f_{s} 8.1$, only two significant loci were identified for the internal eccentricity attributes at $\alpha=0.05$ (Table 1). Both were located on chromosome 2, with ovate as the most significant marker. pei2.1 explained $38 \%$ of the phenotypic variation controlling the internal shape index, and eai 2.1 explained $20 \%$ of the variation controlling eccentricity area index. Overlapping with ovate was the locus pec2.1, pointed shape. controlling proximal eccentricity. Overlapping with $f_{s} 8.1$, the loci pei8.1 and eai8.1 explained $13 \%$ and $10 \%$ of the phenotypic variation and colocalized with this important fruit shape locus (Table 1). Although these QTLs were below the threshold obtained by permutations for a probability $\alpha=0.05$, these loci were significant with the permutation test at the probability of $\alpha=0.1$ (Table 1 ). Thus, ovate and $f_{S} 8.1$ controlled internal shape index and eccentricity area index, whereas ovate also controlled proximal eccentricity.

Fruit from the $\mathrm{SAF}_{2}$ population were also sorted based on their obovoid and widest width position values (Fig. 2D). Similar to the eccentricity attributes, these attributes describe pearshaped fruit and fall under the "asymmetry" function set of TA. We mapped the features in the $\mathrm{SAF}_{2}$ population and found a QTL, ww2.1, on ovate, implying that obovoid and widest width position attributes accurately measure asymmetric fruit (Table 1). However, the threshold significance for the obovoid trait was at a LOD of 22, which indicated that obo2.1 is not significant at the stringent permutation threshold of 0.05 . However, when lowering the stringency to $\alpha=0.1, o b o 2.1$ is significant. Also based on visual observation, the obovoid attribute appeared to accurately measure the degree of pear shape (Fig. 2D). The high permutation threshold for many of the eccentricity and asymmetry attributes was most likely due to the relatively low number of plants in the population that carried pear-shaped fruit (4 of 106). This suggested that the trait is under multigenic control, with ovate having the largest effect in addition to several minor loci. Nevertheless, these attributes in TA offer detailed analysis of shapes that vary from oval to pear to

Initially, the proximal end angles were calculated from the shoulder height and the position of the proximal resulting in one value (Brewer et al., 2006). In the current release of TA version 2.2 , we modified the attribute to measure angles in a fashion similar to measurement for the distal, resulting in several values based on user-defined settings (see "Material and Methods").

Table 5. Correlation matrix of the latitudinal section attributes and tomato fruit weight in the 'Howard German' backcross $\mathrm{F}_{2}$ tomato population.

\begin{tabular}{|c|c|c|c|c|c|c|}
\hline & Pericarp area & Septum + pericarp & Placenta & Lobedness & Locule no. & Fruit weight \\
\hline Pericarp area & 1 & 0.46 & 0.03 & -0.05 & -0.21 & 0.17 \\
\hline Placenta & & & 1 & -0.23 & -0.38 & -0.29 \\
\hline Locule no. & & & & & 1 & 0.44 \\
\hline Fruit weight & & & & & & 1 \\
\hline
\end{tabular}

The significant values $(P<0.01)$ are in bold. 
We compared the results of the two proximal end angles by conducting QTL analyses in the three $\mathrm{F}_{2}$ populations, $\mathrm{HGF}_{2}$, $\mathrm{SAF}_{2}$, and $\mathrm{RGF}_{2}$ (Table 2). The means and standard deviation from TA values were calculated also for the parentals and the $F_{2}$ populations (Table 3). The means of the angles for the $F_{2}$ populations were between the parental values at $2 \%$ and $5 \%$, and higher values were obtained at $10 \%$ and $20 \%$. These values could be due to the effect exerted from the two parental in the genetic control of this trait (Table 2). The results in the $\mathrm{HGF}_{2}$ population showed the same two QTL controlling proximal end angle with the original and improved attribute. One of the QTL corresponded to the sun locus, in addition to one angle QTL on the bottom of chromosome 7 (Table 2). For the $\mathrm{SAF}_{2}$ population, the same two QTL on chromosomes 1 and 2 were found with the original and improved mathematical descriptor for the proximal angle. The locus on chromosome 2 overlapped with ovate (Table 2). An additional proximal end angle QTL was found at $20 \%$ from the top, overlapping with $f_{s} 8.1$. The latter QTL was not found with the original mathematical descriptor for proximal end angle. The proximal end angle analysis of the $\mathrm{RGF}_{2}$ population revealed significant QTLs on chromosomes 1 and 3 (Table 2), of which only the latter was identified with the original mathematical descriptor. Combined, these data indicate that the original and new mathematical descriptors for proximal end angle led to comparable results. Thus, the new proximal end angle descriptor does not invalidate our previous findings. Instead, the new descriptor offers more versatility by allowing the user to measure angles at various positions along the boundary. Moreover, the new proximal end angle attribute is independent from the shoulder height attribute and therefore represents a variable that is independent from other proximal end attributes.

Morphometric analysis. In conjunction with PCA, the morphometric analysis captured the general shape and size by using the points along the fruit boundary. To determine whether morphometric analysis of the fruit shape data resulted in the identification of the same or additional QTL, we compared the individual attribute analyses with the morphometric analyses.

The three $\mathrm{F}_{2}$ populations, $\mathrm{HGF}_{2}, \mathrm{SAF}_{2}$, and $\mathrm{RGF}_{2}$, segregated for elongated fruit shape controlled by the loci sun, ovate, fs8.1, and tri2.1/dblk2.1 (Gonzalo and van der Knaap, 2008). In addition to these four major loci, the shape attributes were controlled by 10 other loci in the tomato genome (Gonzalo and van der Knaap, 2008). We conducted morphometric analyses on the same aforementioned $\mathrm{F}_{2}$ populations. The coordinates were subjected to PCA, which allowed the identification of the major sources of variation. The first three PC collectively explained $87 \%, 96 \%$, and $90 \%$ of the phenotypic variation in $\mathrm{HGF}_{2}, \mathrm{SAF}_{2}$, and $\mathrm{RGF}_{2}$, respectively. Therefore, the first three PC were mapped as QTL. In the $\mathrm{HGF}_{2}$ population, four QTL were detected for $\mathrm{PC} 1$, of which two overlap with shape attribute loci mapped previously (Table 4). The third QTL on chromosome 10 was found to coincide with a fruit size trait that was not reported in the previous study (Gonzalo and van der Knaap, 2008). The morphometric QTL on chromosome 9 was not found in the $\mathrm{HGF}_{2}$ by individual attribute analyses, although it did overlap with a shape QTL in a 'Howard German' backcross population (Brewer et al., 2007). The PC2 trait mapped to two QTL coinciding with the two major fruit shape QTL previously identified in the $\mathrm{HGF}_{2}$ population (Table 4). For example, $p c 2.7$ overlapped with the major fruit shape locus sun and explained $59 \%$ of the phenotypic variance in this population. No significant QTL were detected for the morphometric trait represented by PC3. The morphometric QTL analysis in $\mathrm{SAF}_{2}$ identified two QTL for PC1 and three for PC2. However for the latter PC, two of the three QTL were just below the permutation threshold level at the significance level of 0.05 . When lowering the stringency to 0.1 , both QTLs became significant as the threshold went down to 3.1 and 3.3 respectively. The most significant QTL were $p c 1.11$ and $p c 2.2$ which explained $26 \%$ and $35 \%$ of the phenotypic variance, respectively. The $p c 2.2$ locus overlapped with the major fruit shape locus ovate that was segregating in the $\mathrm{SAF}_{2}$. All of the QTLs detected in $\mathrm{SAF}_{2}$ by morphometric analysis were reported previously for their role in controlling tomato fruit shape (Table 4) (Gonzalo and van der Knaap, 2008). No significant QTL were detected for the morphometric trait represented by PC3. For the third population, four QTL were identified for the $\mathrm{PC} 1$ and $\mathrm{PC} 3$ in the $\mathrm{RGF}_{2}$ population (Table 4), while no significant QTL was found for PC2. All of the QTL were previously reported as regions controlling fruit shape in tomato (Gonzalo and van der Knaap, 2008).

The phenotype of the fruit from the most extreme ends of each PC for which we found significant QTL is shown in Fig. 3. In the $\mathrm{HGF}_{2}$ population, $\mathrm{PC} 1$ showed a distribution of the fruit from round and small to heart-shaped and large, and explained $40 \%$ of the variation. The notion that $\mathrm{HGF}_{2} \mathrm{PC} 1$ identified shape as well as size differences was supported by the fact that the morphometric QTL overlapped with loci that control shape and size of the fruit (Table 4). PC2 explained 32\% of the variation and showed a distribution of the fruits from very pointed and elongated to squat and indented at both proximal and distal end (Fig. 3A). PC2 mostly describes differences in shape as the loci controlling PC2 coincided with QTL controlling shape attributes (Table 4). In the $\mathrm{SAF}_{2}$ population, the first two PC explained $83 \%$ of the variation (Fig. 3B). PC1 showed a distribution of the fruit from small to large and explained 58\% of the variation. The attribute QTL that overlapped with the morphometric PC1 QTL supported the finding that PC1 identified size and shape QTL (Table 4). PC2 explained 25\% of the variation and showed a distribution from squat and indented to pear-shaped fruit (Fig. 3B). Finally, in the $\mathrm{RGF}_{2}$ population, PC1 and PC3 explained $81 \%$ of the phenotypic variance. For both $\mathrm{PC}$, the fruit showed a distribution from round to oval shaped (Fig. 3C). Fruit size differences were also represented by PC1.

Overall, the morphometric analyses led to the identification of 15 QTL representing 10 unique regions of the genome that control morphology of the fruit. With the exception of one QTL (pc1.9 in the $\mathrm{HGF}_{2}$ ), all morphometric QTL were identified previously as attribute QTL. However, not all attribute QTL were identified from the morphometric analyses. For example, one highly significant QTL (tri2.1/dblk2.1 in the $\mathrm{RGF}_{2}$ population; Gonzalo and van der Knaap, 2008) was not identified when we mapped the first three morphometric PC as traits. It is possible that the variation controlled by this locus is represented in a PC that contributes less to the variation (PC4 and onwards). However, the failure to identify significant attribute loci in the first three morphometric PC may also be because morphometrics combine size and shape features, resulting in difficulty discerning subtle shape differences when the largest differences are found for fruit size. For populations that differ significantly in size, such as the three $F_{2}$ populations, shape features may not always be detected above the significance threshold level. The 
morphometric analyses confirmed that most, if not all, of the QTL controlling fruit shape and size in tomato were detected by the attributes implemented in the TA application. This result clearly showed that the attributes capture the major sources of fruit shape variation. Yet, these findings also show the utility of morphometric analyses of shape as a quick and efficient investigation into the morphological variation of the objects under study. As these two methods are available in the same software application, the user can choose to analyze morphological variation quickly using the morphometrics function, followed by more detailed analyses using attribute function.

LATITUDinAL SECTION. Features such as pericarp or placenta area are difficult to capture computationally. Moreover, these features are not based on the outer boundary of the fruit and therefore could not be used in morphometric studies. The attributes that were implemented to measure variation in these traits using cross sections of tomato fruit are under the "latitudinal section" function set of TA. The population that was used to test these features was a selfed 'Howard German' backcross population $\left(\mathrm{HGBC}_{1} \mathrm{~F}_{2}\right)$ population (Fig. 4). For pericarp and pericarp plus septum area, the lowest values were found in fruit with the thinnest pericarp and septum, while the highest values were found in fruit with the thickest pericarp and septum (Fig. 4, A and B). For placenta area, the lowest and highest values are found for fruit with smaller and larger placenta respectively (Fig. 4C). Lastly, for lobedness degree, the most unevenly shaped fruit display the greatest value for this attribute, whereas the most rounded shaped fruit displays the smallest value (Fig. 4D).

The expectation was that pericarp, septum, and pericarp, placenta area, lobedness degree, and locule number were features that could underlie increased fruit size. Indeed, correlation analysis indicated that fruit weight was significantly correlated to all these traits, with the exception of pericarp area (Table 5). We conducted analysis of variance with the markers as groupings and the traits as dependent variables as opposed to linkage mapping because of population structure $\left(\mathrm{BC}_{1} \mathrm{~F}_{2}\right)$ and the incomplete genome-wide marker coverage. We found a significant association of marker SSR162 and the attribute pericarp and septum area (Table 6). For placenta area, the markers $f w 2.2$ on chromosome 2, SSR344 on chromosome 8, and SSR 318 on chromosome 10 were significantly associated with the trait (Table 6). The markers located on chromosome 2 and 10 overlap with fruit size QTLs identified in populations derived from crosses of HG. Moreover, the association of $f w 2.2$ with placenta area was in agreement with previously reported results that indicated that $f w 2.2$ controlled cell division in placental tissues (Liu et al., 2003) and FW2.2 gene expression in placental tissues (Cong et al., 2002). For lobedness, fw2.2 was also the most significant marker in addition to two other markers located on chromosome 7 and 11 (Table 6). The lobedness degree locus on chromosome 7 corresponded to sun, whose main effect is on fruit elongation (van der Knaap and Tanksley, 2001), whereas the lobedness degree locus on chromosome 11 coincided with $f$, which controls locule number and a fruit mass QTL (Barrero and Tanksley, 2004). The attribute locule number was highly significantly controlled by lc (Barrero et al., 2006) near the marker Lewus with a minor effect of $f$ near marker TG393, overlapping with a fruit mass QTL (Table 6). In general, for the attributes that were significantly correlated to fruit mass, two QTL for both weight and the attribute overlapped (Table 6). This indicated that the
Table 6. Results of the ANOVA performed in the 'Howard German' backcross $\mathrm{F}_{2}$ tomato population for the latitudinal section attributes.

\begin{tabular}{lllll}
\hline Attribute & Locus & $\begin{array}{c}\text { Most } \\
\text { significant }\end{array}$ & Corresponding \\
\hline $\begin{array}{c}\text { Pericarp and } \\
\text { septum area }\end{array}$ & sep5.1 & SSR162 & 0.03 & fruit size QTL \\
Placenta area & pla2.1 & fw2.2 & 0.0009 & fw2.2, per2.1, ar2.1 \\
& pla8.1 & SSR344 & 0.0008 & \\
& pla10.1 & SSR318 & 0.009 & per10.1 \\
Lobedness & lob2.1 & fw2.2 & 0.005 & fw2.2, per2.1, ar2.1 \\
degree & & & & \\
& lob7.1 & sun & 0.04 & \\
& lob11.1 & TG36/f & 0.05 & fw11.1 \\
Locule no. & lcn2.1 & Lewus/1c & $<0.0001$ & \\
& lcn3.1 & T0581 & 0.05 & per3.1, ar3.1 \\
& lcn9.1 & cosi52 & 0.02 & \\
& lcn11.1 & TG393/f & 0.007 & fw11.3 \\
\hline
\end{tabular}

latitudinal attributes in TA permitted the identification of the underlying mechanism of increased fruit mass (e.g., increased placenta area or locule number). Moreover, the overlap in locule number and fruit mass QTL might indicate that those QTL underlie the same gene.

Conclusion. We investigated the genetic basis of tomato fruit morphology by subjecting the morphometric output from scanned fruit in segregating populations to quantitative trait analyses. The resulting morphometric QTL were compared with previously identified trait attribute QTL and showed significant overlap. This indicated that both methods were appropriate in identifying regions of genomes that control the variation in morphology. Both methods were implemented in TA, making this tool versatile to researchers interested in a quick assessment of the variation to detailed analysis of a particular shape. We also expanded and improved the TA software tool to support the analysis of morphological features inside the fruit (e.g., pericarp and placenta area). These new features were tested in tomato populations and showed their utility by resulting in the identification of QTL controlling the attributes.

\section{Literature Cited}

Barrero, L.S. and S.D. Tanksley. 2004. Evaluating the genetic basis of multiple-locule fruit in a broad cross section of tomato cultivars. Theor. Appl. Genet. 109:669-679.

Barrero, L.S., B. Cong, F. Wu, and S.D. Tanksley. 2006. Developmental characterization of the fasciated locus and mapping of arabidopsis candidate genes involved in the control of floral meristem size and carpel number in tomato. Genome 49:991-1006.

Bookstein, F.L. 1982. Foundations of morphometrics. Annu. Rev. Ecol. Syst. 13:451-470.

Borba, E.L., R.R. Funch, P.L. Ribeiro, E.C. Smidt, and V. SilvaPereira. 2007. Demography, and genetic and morphological variability of the endangered Sophronitis sincorana (Orchidaceae) in the Chapada Diamantina, Brazil. Plant Syst. Evol. 267:129-146.

Brewer, M.T., J.B. Moyseenko, A.J. Monforte, and E. van der Knaap. 2007. Morphological variation in tomato: A comprehensive study of quantitative trait loci controlling fruit shape and development. J. Expt. Bot. 58:1339-1349.

Brewer, M.T., L. Lang, K. Fujimura, N. Dujmovic, S. Gray, and E. van der Knaap. 2006. Development of a controlled vocabulary and 
software application to analyze fruit shape variation in tomato and other plant species. Plant Physiol. 141:15-25.

Chandler, G.T. and M.D. Crisp. 1998. Morphometric and phylogenetic analysis of the Daviesia ulicifolia complex (Fabaceae, Mirbeliae). Plant Syst. Evol. 209:93-112.

Cheverud, J.M. 1996. Developmental integration and the evolution of pleiotropy. Amer. Zool. 36:44-50.

Churchill, G.A. and R.W. Doerge. 1994. Empirical threshold values for quantitative trait mapping. Genetics 138:963-971.

Cong, B., J. Liu, and S.D. Tanksley. 2002. Natural alleles at a tomato fruit size quantitative trait locus differ by heterochronic regulatory mutations. Proc. Natl. Acad. Sci. USA 99:13606-13611.

Dryden, I.L. and K.V. Mardia. 1998. Statistical analysis of shape. Wiley, Chichester, UK.

Gonzalo, M.J. and E. van der Knaap. 2008. A comparative analysis into the genetic bases of morphology in tomato varieties exhibiting elongated fruit shape. Theor. Appl. Genet. 116:647-656.

Henderson, A. 2006. Traditional morphometrics in plant systematics and its role in palm systematics. Bot. J. Linn. Soc. 151:103-111.

Klingenberg, C. and L. Monteiro. 2005. Distances and directions in multidimensional shape spaces: Implications for morphometric applications. Syst. Biol. 54:678-688.

Klingenberg, C.P., L.J. Leamy, E.J. Routman, and J.M. Cheverud. 2001. Genetic architecture of mandible shape in mice: Effects of quantitative trait loci analyzed by geometric morphometrics. Genetics 157:785-802.

Langlade, N.B., X. Feng, T. Dransfield, L. Copsey, A.I. Hanna, C. Thebaud, A. Bangham, A. Hudson, and E. Coen. 2005. Evolution through genetically controlled allometry space. Proc. Natl. Acad. Sci. USA 102:10221-10226.

Lihova, J., K. Marhold, A. Tribsch, and T.F. Stuessy. 2004. Morphometric and AFLP re-evaluation of tetraploid Cardamine amara (Brassicaceae) in the Mediterranean. Syst. Bot. 29:134-146.

Liu, J., B. Cong, and S.D. Tanksley. 2003. Generation and analysis of an artificial gene dosage series in tomato to study the mechanisms by which the cloned quantitative trait locus $f w 2.2$ controls fruit size. Plant Physiol. 132:292-299.
Paran, I. and E. van der Knaap. 2007. Genetic and molecular regulation of fruit and plant domestication traits in tomato and pepper. J. Expt. Bot. 58:3841-3852.

Perez-Perez, J.M., J. Serrano-Cartagena, and J.L. Micol. 2002. Genetic analysis of natural variations in the architecture of arabidopsis thaliana vegetative leaves. Genetics 162:893-915.

Rohlf, F.J. 1990. Morphometrics. Annu. Rev. Ecol. Systematics 21:299-316.

Sonibare, M.A., A.A. Jayeola, and A. Egunyomi. 2004. A morphometric analysis of the genus Ficus (Moraceae). Afr. J. Biotechnol. 3:229-235.

Van der Knaap, E. and S.D. Tanksley. 2001. Identification and characterization of a novel locus controlling early fruit development in tomato. Theor. Appl. Genet. 103:353-358.

Van der Knaap, E., S. Gray, K. Fujimura, L. Lang, N. Dujmovic, D. Sullivan, M. Brewer, M.J. Gonzalo, G. Rodriguez, and C. Anderson. 2008. Tomato Analyzer. 28 July 2008. <http://www.oardc.ohio-state. edu/vanderknaap/TArelease.htm>.

Van der Knaap, E., Z.B. Lippman, and S.D. Tanksley. 2002. Extremely elongated tomato fruit controlled by four quantitative trait loci with epistatic interactions. Theor. Appl. Genet. 104:241247.

Wang, S., C.J. Basten, and Z.B. Zeng. 2006. Windows QTL Cartographer 2.5. 22 July 2008. <http://statgen.ncsu.edu/qtlcart/ WQTLCart.htm>

Weber, K., R. Eisman, L. Morey, A. Patty, J. Sparks, M. Tausek, and Z.B. Zeng. 1999. An analysis of polygenes affecting wing shape on chromosome 3 in Drosophila melanogaster. Genetics 153:773786

Weight, C., D. Parnham, and R. Waites. 2008. Leafanalyser: A computational method for rapid and large-scale analyses of leaf shape variation. Plant J. 53:578-586.

Zeng, Z.B. 1993. Theoretical basis for separation of multiple linked gene effects in mapping quantitative trait loci. Proc. Natl. Acad. Sci. USA 90:10972-10976.

Zeng, Z.B. 1994. Precision mapping of quantitative trait loci. Genetics 136:1457-1468. 